\title{
The impact of COVID-19 on Abu Dhabi and its primary care response
}

\section{Reem Juma Al Falasi, Moien AB Khan}

CoVID-19 constitutes a significant challenge for primary care on a global scale, resulting in a transition from focused individual care to a public health-based response. ${ }^{1}$ In Abu Dhabi, primary care is provided at ambulatory healthcare centres (AHS). The Primary Care Team (PCT) consists of primary care physicians and allied healthcare professionals. ${ }^{2}$ PCTs have responded to curb the spread of the virus by working alongside the government on the frontline by prioritising five key areas outlined in this article. These aim to protect the health, safety and wellbeing of the community in order to ensure the best possible care for the population.

\section{Flattening the curve}

PCTs trained by the National Emergency

Crisis and Disaster Management Authority conduct screening and surveillance in the newly implemented drive-through COVID-19 testing centres across Abu Dhabi. ${ }^{3}$ Furthermore, a targeted testing program in high-risk overcrowded residential and industrial zones to identify active cases is being carried out by PCTs. In addition, PCTs in conjunction with public health authorities are actively educating the public about prevention measures, promoting the use of face masks in public, restricting social gatherings, reiterating the need for social distancing and identifying the clinical signs of COVID-19, all of which have been useful in curtailing the spread.

\section{Improving care delivery to patients with COVID-19}

More than $80 \%$ of patients with COVID-19 have only experienced mild symptoms ${ }^{4}$ and are therefore managed in primary care. Those presenting with milder symptoms are educated, treated symptomatically and advised to self-isolate at home and are subsequently receiving follow-up treatment in COVID-19-dedicated AHS. Smart digital platforms using artificial intelligence to trace people who have been in contact with patients who have COVID-19 are also being used, in the hope that these contacts will be tested. ${ }^{5}$ Patients with existing medical conditions experiencing only mild symptoms are treated virtually, while those who are very unwell are being admitted into the newer field hospitals. Furthermore, counselling and virtual support are provided to the patients by primary care counsellors.

\section{Ensuring continuity of care}

Patients with comorbidities are avoiding or delaying their care routines during the pandemic, which is placing them at a greater risk of developing complications. ${ }^{6}$ Newer care delivery models in the form of remote healthcare platforms have been implemented in primary care clinics. PCTs assist in managing the health of such patients by contacting them via phone and through online video consultations. Medications are then delivered to their homes by pharmacies. Patients that need to be seen are being received in selected drive-through clinics for examination, investigation and treatment by the PCTs. The patient appreciation and positive impact of these services has been evident and has been reflected in the patient feedback. Virtual counselling has been useful and popular with the patients and is being provided through dedicated counsellors within the PCTs.

\section{Streamlining capacity of the healthcare centres}

Most of the primary care consultations occur via telephone and video consultations. Those who are unwell or who need urgent treatment are managed at designated healthcare centres. Drive-through child vaccination services are also being provided. Preventive screenings are done through telephone consultations, followed by a review at the primary care clinic if needed. The healthcare centres have been reorganised to minimise time patients spend in the clinic should they require a physical examination.

\section{Protecting the physical and mental health of healthcare workers}

Adequate personal protective equipment is being provided, and PCTs are supported through regular communication channels. Each member of the healthcare team is tested for COVID-19 every two weeks and isolated from work if positive or symptomatic. With regards to addressing the psychological strain caused by the COVID-19 pandemic and the inevitable 
increase in workload, the PCTs are supported by counselling and support helplines through the SEHA Employees Psychological Support Taskforce (SEPST). ${ }^{7}$ In addition, weekly psychological motivational webinars, in conjunction with positive weekly team challenges, encourage inter-staff communication and allow staff to appreciate and encourage each other's work.

Thus, to conclude, the role of PCTs in Abu Dhabi has been essential and they have made a valuable contribution to the population in managing COVID-19, preventing other diseases from spreading and providing continuous care to those with existing health conditions. With a rapid response to the changing dynamics of the pandemic, a combination of evidence-based decisions and collaboration with the PCTs in Abu Dhabi is providing an efficient level of care and is playing a major role in both the medical and psychological care of the population during this unprecedented pandemic.

\section{First published online 8 September 2020.}

\section{Authors}

Reem Juma AI Falasi MBBS, MSc (LHPE), Arab Board (Family Medicine), Family Physician and Health Center Manager, Al Maqam Healthcare Center, Ambulatory Healthcare Services, Abu Dhabi, United Arab Emirates; Adjunct Assistant Professor, Department of Family Medicine, College of Medicine and Health Sciences, United Arab Emirates University, Abu Dhabi, United Arab Emirates

Moien AB Khan MBBS, MSc (Diabetes), MSc (HEPM), MRCGP - CCT (UK), Chair, Department of Family Medicine, College of Medicine and Health Sciences, United Arab Emirates University, Abu Dhabi, United Arab Emirates; Consultant Family Physician, Ambulatory Healthcare Services, Abu Dhabi, United Arab Emirates. moien.khan@uaeu.ac.ae

Competing interests: None.

Provenance and peer review: Commissioned, peer reviewed.

Citation: Al Falasi RJ, Khan MAB. The impact of COVID-19 on Abu Dhabi and its primary care response. Aust J Gen Pract 2020;49 Suppl 35. doi: 10.31128/AJGP-COVID-35.

\section{References}

1. Majeed A, Maile EJ, Bindman AB. The primary care response to COVID-19 in England's National Health Service. J R Soc Med 2020;113(6):208-10. doi: 10.1177/0141076820931452.

2. Paulo MS, Loney T, Lapão LV. The primary health care in the emirate of Abu Dhabi: Are they aligned with the chronic care model elements? BMC Health Serv Res 2017;17(1):725. doi: 10.1186/ s12913-017-2691-4.
3. Vally Z. Public perceptions, anxiety, and the perceived efficacy of health-protective behaviours to mitigate the spread of the SARSCov-2/COVID-19 pandemic. Public Health 2020. doi: 10.1016/j.puhe.2020.08.002.

4. Wu Z, McGoogan JM. Characteristics of and important lessons from the coronavirus disease 2019 (COVID-19) outbreak in China: Summary of a report of 72314 cases from the Chinese center for disease control and prevention. JAMA 2020. doi: 10.1001/jama.2020.2648.

5. U.ae. Smart solutions to fight COVID-19. Abu Dhabi, UAE: Telecommunications Regulatory Authority, 2020. Available at https://u.ae/en/ information-and-services/justice-safety-andthe-law/handling-the-covid-19-outbreak/ smart-solutions-to-fight-covid-19 [Accessed 3 September 2020]

6. Ziade N, el Kibbi L, Hmamouchi l, et al. The impact of the COVID-19 pandemic on patients with chronic rheumatic diseases: A study in 15 Arab countries. Rheumatology 2020. doi: 10.21203/ rs.3.rs-40892/v1.

7. Tohme W, Schmitz-Hübsch J, Ahmed N. How GCC governments can create resilient mental health services. Omina Health. 30 June 2020. Available at https://insights.omnia-health.com/ coronavirus-updates/how-gcc-governments-cancreate-resilient-mental-health-services [Accessed 3 September 2020]. 